\title{
Avaliação da presença de biotoxinas marinhas em moluscos bivalves na região da Costeira do Ribeirão em Florianópolis, Santa Catarina
}

\author{
Evaluation of the presence of marine biotoxins in bivalve molluscs in the coastal region of \\ Ribeirão in Florianópolis, Santa Catarina \\ L. Pigozzi*; V. S. Freitas; V. A. Salazar; S. Verruck \\ Departamento de Ciência e Tecnologia de Alimentos, Universidade Federal de Santa Catarina, 88040-900, \\ Florianópolis-SC, Brasil \\ *luizabepgzz@gmail.com \\ (Trabalho avaliado e selecionado pela Comissão do III CTOCTA)
}

\begin{abstract}
Santa Catarina representa a maior parte da produção de moluscos bivalves no Brasil e sua capital, Florianópolis, é um grande polo de cultivo sendo a Costeira do Ribeirão da Ilha, bairro localizado no sul da ilha, um local de grande produtividade. O presente trabalho teve como objetivo avaliar os resultados das análises de monitoramento de ficotoxinas realizadas em ostras e mexilhões provenientes da Costeira do Ribeirão da Ilha no período de outubro de 2019 até outubro de 2020. A compilação dos dados demonstrou ocorrência de biotoxinas marinhas na região em cinco datas. Os laudos apresentaram valores de ácido

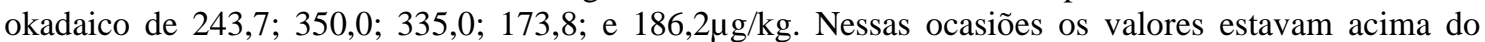
máximo permitido pela legislação brasileira e, por isso, a retirada e comercialização dos moluscos foi proibida temporariamente no período em que as contaminações estavam presentes. Sendo, assim, este trabalho demonstra que o monitoramento da qualidade das águas e moluscos bivalves têm grande importância para garantir a saúde do consumidor, tendo em vista a tendência de expansão do cultivo e consumo desse alimento nos próximos anos.

Palavras-chave: ficotoxinas, ácido okadaico, mexilhões.
\end{abstract}

Santa Catarina represents the major part of the production of bivalve mollusks in Brazil. Its capital, Florianópolis, is a big cultivation pole and the coastal area of Ribeirão da Ilha, a neighborhood located in the south of the island, is a place with significant production. The present study aimed to evaluate the results of the monitoring analysis of phytotoxins carried out on oysters and mussels from the coastal area of Ribeirão da Ilha, from October 2019 to October 2020. The compilation of data showed the occurrence of marine biotoxins in the region on five dates. The reports presented okadaic acid values of $243.7 ; 350.0$; $335.0 ; 173.8$; and $186.2 \mu \mathrm{g} / \mathrm{kg}$. On these occasions, the values were above the maximum allowed by Brazilian legislation and, therefore, the removal and commercialization of mollusks were temporarily prohibited in the period when the contamination was present. Thus, this work demonstrates that monitoring the quality of water and bivalve mollusks are of great importance to ensure consumer's health, having in mind the possible expansion of the cultivation and consumption of this kind of food in the coming years. Keywords: phytotoxins, okadaic acid, mussels.

\section{INTRODUÇÃO}

A aquicultura é a criação de animais aquáticos, incluindo peixes, crustáceos, moluscos e plantas aquáticas cultivadas em água doce ou água do mar. Em 2018, a aquicultura mundial atingiu o recorde com a produção de 114,5 milhões de toneladas de peso vivo, sendo que 18 milhões de toneladas correspondem a produção de moluscos [1]. No Brasil, o estado de Santa Catarina destaca-se como o grande produtor de moluscos bivalves, responsável pelo cultivo de 95\% do total produzido no país. Segundo o Centro de Desenvolvimento de Agricultura e Pesca (CEDAP) de Santa Catarina, no ano de 2019 foram produzidas 13.561 toneladas de moluscos envolvendo 485 malacocultores, dos quais os principais polos de produção foram os municípios de Palhoça, Florianópolis e Bombinhas, gerando mais de 1.900 empregos diretos [2]. O Laboratório de Moluscos Marinhos da Universidade Federal de Santa Catarina (LMM/UFSC) é o principal produtor de sementes de moluscos, que são entregues a maricultores em todo o Brasil, representando mais de 30 milhões de sementes produzidas [3]. 
Florianópolis é a capital catarinense popularmente conhecida pelas praias e pela culinária local, fortemente caracterizada pela presença de frutos do mar. Dividida em baía norte e sul, a cidade apresenta diversas localidades produtoras de moluscos bivalves e a Costeira do Ribeirão da Ilha é um local de grande produção. Essa localidade está situada na parte sul da ilha e é habitada principalmente por famílias que tem como principal fonte de renda a malacocultura, fazendo com que a economia local gire em torno do cultivo e comercialização de ostras e mexilhões [4].

Segundo o Instituto Português do Mar e da Atmosfera (IPMA), molusco bivalve é o termo que designa o animal de corpo mole protegido por um exoesqueleto com forma de concha. A respiração e alimentação destes animais se dá por meio da filtragem de grande quantidade de água e, por isso, eles podem reter no seu interior moléculas e substâncias provenientes do meio externo [5]. Essa característica exige que o controle da qualidade da água seja feito rigorosamente para garantir a qualidade e segurança do alimento. Dentre as substâncias que podem ser filtradas, estão as biotoxinas marinhas que, segundo a Instrução Normativa Interministerial $\mathrm{n}^{\circ} 7$, de 8 de maio de 2012, são definidas como toxinas termoestáveis, provenientes de fitoplâncton, que, quando ingeridas pelo homem, podem levar a sérias complicações de saúde. Existem diversas síndromes associadas ao consumo de moluscos bivalves contaminados, podendo ser divididas em cinco grandes grupos: Intoxicação paralisante - PSP, Intoxicação diarreica - DSP, Intoxicação amnésica - ASP, Intoxicação neurológica - NSP, Intoxicação por consumo de azaspirácidos - AZP [6].

Para garantir a segurança e qualidade dos moluscos bivalves, a Companhia Integrada de Desenvolvimento Agrícola de Santa Catarina [7] executa análises quinzenais em amostras de águas e moluscos para verificar a presença ou não de biotoxinas marinhas. Neste contexto, o presente trabalho visa coletar dados sobre essas análises, discutir e justificar as informações encontradas com base na literatura.

\section{MATERIAL E MÉTODOS}

O presente trabalho foi realizado através da coleta de dados a partir dos laudos oficiais disponibilizados no site da Companhia Integrada de Desenvolvimento Agrícola de Santa Catarina (CIDASC) [8, 9]. Foi feita a compilação dos resultados de análises físico-químicas realizadas para verificar a ocorrência de biotoxinas marinhas em mexilhões e ostras na localidade da Costeira do Ribeirão da Ilha, em Florianópolis (Figura 1), Santa Catarina. O período considerado foi de outubro de 2019 a outubro de 2020.
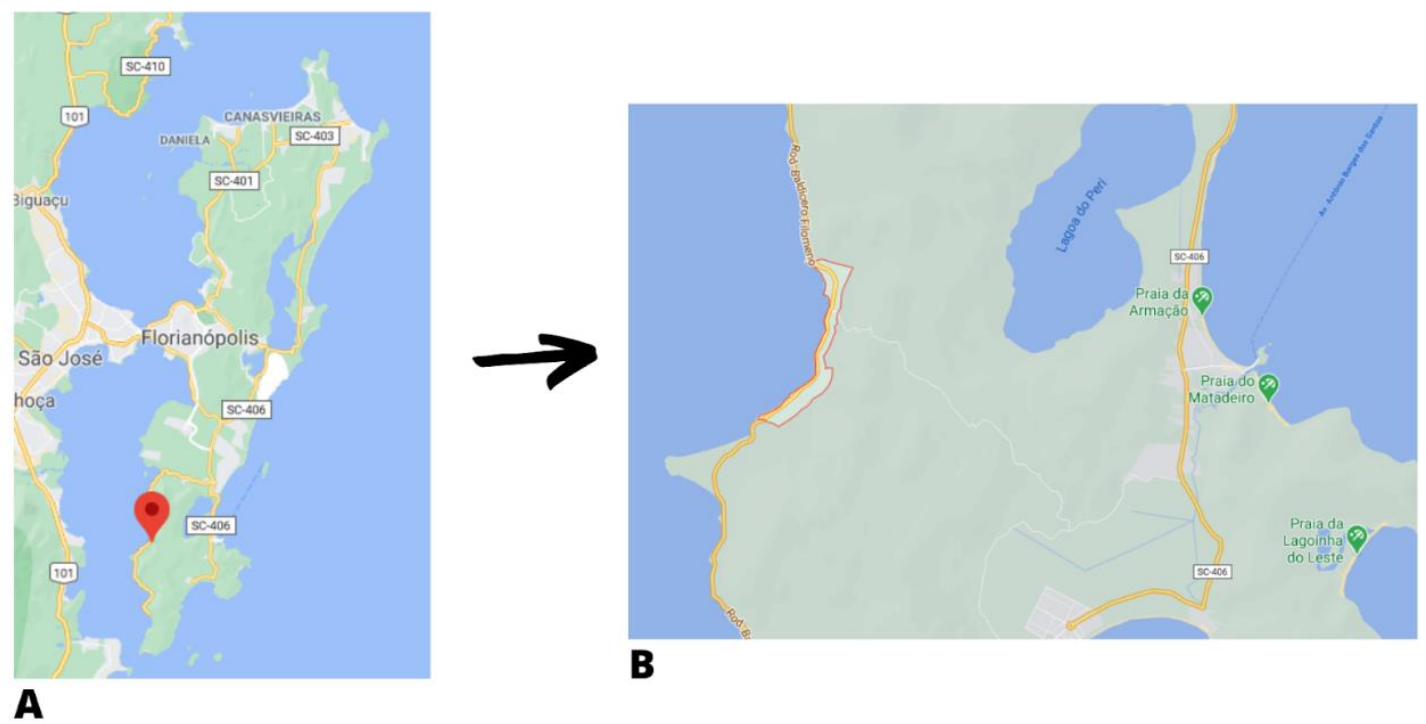

Figura 1: A - mapa da ilha de Florianópolis/SC. B - mapa da região estudada, Costeira do Ribeirão da Ilha, delimitada pela linha vermelha. Fonte: Google Maps. 
Vale salientar que o método utilizado para quantificação de biotoxinas marinhas durante o monitoramento é baseado na metodologia de Molognoni et al. (2019) [10], sendo a cromatografia líquida acoplada à espectrometria de massas (LC-MS / MS) com ionização por electrospray (ESI) nos modos positivo e negativo a metodologia utilizada para os ensaios de detecção e quantificação de ficotoxinas em moluscos bivalves [10].

\section{RESULTADOS E DISCUSSÃO}

As análises laboratoriais para acompanhamento da presença de biotoxinas marinhas em moluscos bivalves são de extrema importância para a saúde humana e para a segurança de alimentos. Esses compostos não prejudicam diretamente o molusco em si, podendo dizer que eles são imunes aos efeitos diretos uma vez que não há danos ao tecido animal. Entretanto, são considerados veículos para intoxicação de seres humanos, podendo desencadear uma série de problemas à saúde de seus consumidores [11].

Para que o consumo de moluscos bivalves seja considerado seguro, é preciso que eles respeitem os limites máximos de biotoxinas designados pela Instrução Normativa Interministerial $\mathrm{n}^{\mathrm{o}} 7$, de 8 de maio de 2012 [6]. Na Tabela 1 são apresentados os limites permitidos de biotoxinas produzidas por microalgas em $1 \mathrm{~kg}$ da parte comestível dos moluscos bivalves [6].

Tabela 1: Limites máximos permitidos de biotoxinas marinhas presentes em moluscos bivalves para sua retirada e comercialização [6].

\begin{tabular}{rccccc}
\cline { 2 - 6 } Ficotoxina & $\begin{array}{c}\text { Ácido } \\
\text { domoico }\end{array}$ & $\begin{array}{c}\text { Equivalente à } \\
\text { saxitoxina }\end{array}$ & $\begin{array}{c}\text { Equivalente à } \\
\text { yessotoxina }\end{array}$ & $\begin{array}{c}\text { Equivalente ao } \\
\text { ácido okadaico }\end{array}$ & $\begin{array}{c}\text { Equivalente aos } \\
\text { azaspirácidos }\end{array}$ \\
\hline Máximo & $20 \mathrm{mg} / \mathrm{kg}$ & $800 \mu \mathrm{g} / \mathrm{kg}$ & $1 \mathrm{mg} / \mathrm{kg}$ & $160 \mu \mathrm{g} / \mathrm{kg}$ & $60 \mu \mathrm{g} / \mathrm{kg}$ \\
\hline
\end{tabular}

A apresentação dos dados de avaliação de biotoxinas marinhas realizadas na Costeira do Ribeirão da Ilha no período de outubro de 2019 a outubro de 2020 estão dispostos na Tabela 2. Apenas os laudos com resultados positivos foram apresentados na Tabela 2, porém vale salientar que o monitoramento ocorre quinzenalmente. Diante dos resultados, foi detectada a ocorrência da presença da ficotoxina ácido okadaico na região da Costeira do Ribeirão em três dos doze meses analisados. Dos 56 laudos analisados, somente cinco análises de mexilhões apresentaram valores desta ficotoxina acima do máximo permitido, ou seja, $186.2 \mu \mathrm{g} / \mathrm{Kg}$ em agosto, 173.8, 335.0, 350.0 e $243.7 \mu \mathrm{g} / \mathrm{Kg}$ em outubro de 2020 , visto que o valor máximo permitido pela legislação vigente é de $160 \mu \mathrm{g} / \mathrm{Kg}[6]$.

Tabela 2: Ocorrência de ficotoxinas em ostras e mexilhões vivos na localidade da Costeira do Ribeirão, em Florianópolis/SC, entre outubro de 2019 a outubro de 2020.

\begin{tabular}{|c|c|c|c|c|c|c|}
\hline $\begin{array}{l}\text { Data da } \\
\text { coleta }\end{array}$ & Molusco & $\begin{array}{c}\text { Ácido } \\
\text { domoico } \\
(\mathrm{mg} / \mathrm{kg})\end{array}$ & $\begin{array}{c}\text { Equivalente } \\
\text { à saxitoxina } \\
(\mu \mathrm{g} / \mathrm{kg})\end{array}$ & $\begin{array}{c}\text { Equivalente à } \\
\text { yessotoxina } \\
(\mathrm{mg} / \mathrm{kg})\end{array}$ & $\begin{array}{c}\text { Equivalente ao } \\
\text { ácido okadaico } \\
(\mu \mathrm{g} / \mathrm{kg})\end{array}$ & $\begin{array}{c}\text { Equivalente ao } \\
\text { azaspirácido } \\
(\mu \mathrm{g} / \mathrm{kg})\end{array}$ \\
\hline \multirow[t]{2}{*}{$10 / 2020$} & Ostra & NR & NR & NR & 85,0 & NR \\
\hline & Mexilhão & NR & NR & NR & 243,7 & NR \\
\hline \multirow[t]{2}{*}{$10 / 2020$} & Ostra & NR & NR & NR & 80,0 & NR \\
\hline & Mexilhão & NR & NR & NR & 350,0 & NR \\
\hline \multirow{2}{*}{$10 / 2020$} & Ostra & NR & NR & NR & 152,5 & NR \\
\hline & Mexilhão & NR & NR & NR & 335,0 & NR \\
\hline \multirow{2}{*}{$10 / 2020$} & Ostra & NR & NR & NR & $<40$ & NR \\
\hline & Mexilhão & NR & NR & NR & 173,8 & NR \\
\hline \multirow{2}{*}{ 09/2020 } & Ostra & ND & ND & NR & 50,0 & ND \\
\hline & Mexilhão & ND & ND & NR & 88,7 & ND \\
\hline
\end{tabular}


Tabela 2: (continuação)

\begin{tabular}{lclllcl}
\hline \multirow{208/2020}{*}{} & Ostra & ND & ND & NR & 82,4 & ND \\
& Mexilhão & ND & ND & NR & 108,8 & ND \\
$\mathbf{0 8 / 2 0 2 0}$ & Ostra & ND & ND & NR & 67,4 & ND \\
& Mexilhão & ND & ND & NR & $\mathbf{1 8 6 , 2}$ & ND \\
\hline
\end{tabular}

NR: Não realizado. ND: Não detectado. Limite de Quantificação do método: Ácido domoico LQ 0,5mg/kg; à

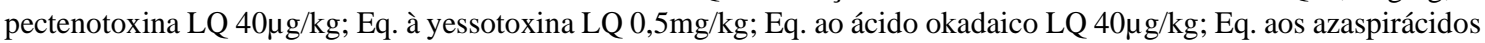
LQ $40 \mu \mathrm{g} / \mathrm{kg}$;.Incerteza padrão expandida relativa $(\mathrm{k}=2,00)$ na tomada de decisão no LR: equivalentes ao ácido okadaico $30 \%$; equivalentes aos azaspirácidos $24 \%$; equivalentes à yessotoxina $8 \%$; ácido domóico $2 \%$ e equivalentes à saxitoxina 24\%. Método de análise: MET POA_SLAV/061/0 [10].

Ao analisar os dados, pode-se inferir que o ácido okadáico é a biotoxina encontrada mais corriqueiramente na costa catarinense e pode ser considerada a causa mais comum de interdição de locais de cultivo de moluscos bivalves. Essa toxina atua no organismo humano como inibidor potente de uma classe de enzimas - as fosfatases proteicas, desregulando diversos processos metabólicos provocando sintomas como náuseas, vômitos e dores abdominais, sendo raros quadros mais graves e necessidade de hospitalização [12]. Sendo assim, quando as análises laboratoriais constatam a extrapolação dos limites de biotoxinas previstos na legislação, a retirada e comercialização dos moluscos é temporariamente proibida até que ocorra a depuração natural das biotoxinas pelos moluscos, visando assegurar a saúde do consumidor.

Conforme os dados coletados, houveram duas interdições na Costeira do Ribeirão da Ilha durante o período analisado neste estudo. A primeira interdição ocorreu no dia 6 de agosto quando foi detectado $186,2 \mu \mathrm{g} / \mathrm{Kg}$ de ácido okadaico nas amostras de mexilhões coletadas. Após o segundo resultado negativo consecutivo a área pôde voltar a retirar e comercializar os moluscos normalmente [13]. A segunda interdição foi no dia 9 de outubro após a detecção de $173,8 \mu \mathrm{g} / \mathrm{Kg}$ de ácido okadaico, também em amostras de mexilhões. No fim do mês de outubro, as análises foram repetidas e os resultados mostraram que a concentração da biotoxina continuou acima do limite permitido para os mexilhões enquanto que a concentração da toxina nas ostras não excedeu os limites impostos pela legislação vigente. Com isso, houve a liberação parcial do cultivo dos moluscos bivalves [14]. A liberação parcial consiste em permitir a coleta e comercialização somente de uma parte do cultivo. Neste caso, as ostras seguem sendo produzidas e coletadas normalmente devido à baixa concentração de biotoxina enquanto que os mexilhões permanecem impedidos até que sejam analisados novamente. A ostra concentra menos toxinas e, por isso, foi possível a sua liberação antes dos mexilhões [15].

É importante ressaltar que assim como as demais toxinas, o ácido okadaico é termoestável, ou seja, mesmo que os moluscos sofram processamento térmico as ficotoxinas não são degradadas, sendo prudente e necessária a interdição da área de cultivo. Embora essa toxina não provoque sintomas irreversíveis, é obrigação dos malacocultores e de todos os envolvidos na cadeia produtiva garantir que os moluscos cheguem à mesa do consumidor final de forma segura e com qualidade [16].

O acompanhamento da qualidade dos moluscos bivalves é de extrema importância, sendo que o Programa Estadual de Controle Higiênico-Sanitário de Moluscos Bivalves - PNCMB, executado pela CIDASC, é essencial para a gestão e controle sanitário da cadeia produtiva dos moluscos. O monitoramento ganha tamanha importância uma vez que a floração das microalgas produtoras das toxinas ocorre naturalmente, sendo que as condições e a própria produção das ficotoxinas não é totalmente conhecida pela ciência, o que torna imprescindível esse monitoramento constante por parte dos órgãos fiscalizadores [7].

Há estudos que visam melhorar a metodologia de monitoramento e que buscam determinar os fatores que levam a uma maior ocorrência de microalgas produtoras de biotoxinas marinhas em ocasiões específicas. Assim, será mais fácil de prever as condições em que ocorrem as florações dessas algas para buscar os cenários em que esse fenômeno acontece com mais frequência, facilitando as tomadas de decisão e gestão da cadeia produtiva [16]. 


\section{CONCLUSÃO}

Diante do exposto, pode-se concluir que a Costeira do Ribeirão da Ilha apresentou incidência de um grupo de ficotoxinas, o ácido okadaico ao longo do período de novembro de 2019 a novembro de 2020. Sendo assim, ressalta-se a importância do monitoramento contínuo das águas e moluscos bivalves para garantir a saúde e segurança do consumidor.

O consumo de frutos do mar está crescendo com o passar dos anos e conforme relatório da FAO, o Brasil provavelmente aumentará em $104 \%$ a sua produção de pesca e aquicultura em 2025. Com essa grande e importante oportunidade de expansão da produção, são necessários mais investimento e estudo no setor para a melhoria constante dos processos de produção, monitoramento e análise dos moluscos e a implementação de projetos que visem estimular e apoiar a malacocultura local.

\section{AGRADECIMENTOS}

Agradecemos à Universidade Federal de Santa Catarina pelo apoio no presente trabalho.

\section{REFERÊNCIAS BIBLIOGRÁFICAS}

1. Food and Agriculture Organization of the United Nations (FAO). The state of world fisheries and aquaculture 2020. Sustainability in action [Internet]; 2020 [citado em 12 out 2020]. Disponível em: http://www.fao.org/state-of-fisheries-aquaculture

2. Empresa de Pesquisa Agropecuária e Extensão Rural de Santa Catarina (EPAGRI). Números da agropecuária catarinense - 2020 [Internet]. Florianópolis (SC): Epagri; 2020 [citado em 08 nov 2020]. Disponível em: https://publicacoes.epagri.sc.gov.br/DOC/article/view/1067/955

3. Albuquerque MCP. Laboratório de Moluscos Marinhos da UFSC mantém produção durante a pandemia. Notícias da UFSC [Internet]; 22 jul 2020 [citado em 29 out 2020]. Disponível em: https://noticias.ufsc.br/2020/07/laboratorio-de-moluscos-marinhos-da-ufsc-mantem-producao-durantea-pandemia/

4. Governo de Santa Catarina, Epagri / Cedap [Internet]. Maricultura; c1996-2021 [citado em 20 out 2020]. Disponível em: https://cedap.epagri.sc.gov.br/index.php/maricultura/

5. Silva HA, Costa P, Rodrigues S. Morfologia, biologia e ecologia dos moluscos bivalves. Instituto Português do Mar e da Atmosfera - IPMA [Internet]; c2020 [citado em 20 out 2020]. Disponível em: https://www.ipma.pt/pt/enciclopedia/pescas/index.html\#: :text=0\%20termo\%20molusco\%20bivalve

6. Brasil. Ministério da Pesca e Aquicultura. Instrução Normativa Interministerial MPA/MAPA n ${ }^{\circ}$, de 08 de maio de 2012. Institui o Programa Nacional de Controle Higiênico-Sanitário de Moluscos Bivalves (PNCMB), estabelece os procedimentos para a sua execução e dá outras providências. Diário Oficial da União. 09 mai 2012;89(Seção 1):55. Disponível em: https://www.gov.br/agricultura/ptbr/assuntos/aquicultura-e-pesca/legislacao/legislacao-geral-da-pesca/ini-mpa-mma-ndeg-7-de-08-052012.pdf/view

7. Ascom JV. Santa Catarina é destaque na produção nacional de moluscos bivalves. CIDASC: Companhia Integrada de Desenvolvimento Agrícola de Santa Catarina [Internet]; 07 jun 2020 [citado em 19 out 2020]. Disponível em: http://www.cidasc.sc.gov.br/blog/2020/06/07/santa-catarina-e-destaque-naproducao-nacional-de-moluscos-bivalves/

8. CIDASC [Internet]. Defesa sanitária animal. Resultado do monitoramento de algas nocivas e ficotoxinas em moluscos bivalves - 2019; 2019 [citado em 09 out 2020]. Disponível em: http://www.cidasc.sc.gov.br/defesasanitariaanimal/resultado-do-monitoramento-de-algas-nocivas-eficotoxinas-em-moluscos-bivalves-2019/

9. CIDASC [Internet]. Defesa Sanitária Animal. Resultado do monitoramento de algas nocivas e ficotoxinas em moluscos bivalves - 2020; 2020. [citado em 09 out 2020]. Disponível em: http://www.cidasc.sc.gov.br/defesasanitariaanimal/resultado-do-monitoramento-de-algas-nocivas-eficotoxinas-em-moluscos-bivalves-2020/

10. Molognoni L, Dos Santos JN, Kleemann CR, Costa ACO, Hoff RB, Daguer H. Cost-effective and highreliability analytical approach for multitoxin screening in bivalve mollusks by liquid chromatography coupled to tandem mass spectrometry. J Agric Food Chem. 2019;67(9):2691-9. doi: $10.1021 /$ acs.jafc. 8 b06600

11. Mariné GF, Silva PPO, Oliveira GM, Ferreira VM. Detecção de ácido ocadaico em cultivo de mexilhões Perna perna, Angra dos Reis, RJ. Ciência Rural. 2010;40(1):193-6. 
12. Vale P. Biotoxinas marinhas - Marine biotoxins. Rev Portuguesa Med Veterinária. 2004;99(549):3-18.

13. Liberada a venda de moluscos da Freguesia do Ribeirão e Costeira do Ribeirão, em Florianópolis. Secretaria de Estado da Agricultura, da Pesca e do Desenvolvimento Rural [Internet]; 21 ago 2020 [citado em 08 nov 2020]. Disponível em: https://www.agricultura.sc.gov.br/index.php/noticias/1230liberada-a-venda-de-moluscos-da-freguesia-do-ribeirao-e-costeira-do-ribeirao-em-florianopolis

14. Secretaria da Agricultura anuncia interdição em cultivos de moluscos de Florianópolis. Secretaria de Estado da Agricultura, da Pesca e do Desenvolvimento Rural [Internet]; 23 out 2020 [citado em 08 nov 2020] Disponível em: https://www.agricultura.sc.gov.br/index.php/noticias/1268-secretaria-daagricultura-anuncia-interdicao-de-areas-de-cultivo-de-moluscos-em-florianopolis

15. Secretaria da Agricultura atualiza situação dos cultivos de moluscos em Santa Catarina. Governo de Santa Catarina [Internet]; 29 out 2020 [citado em 08 nov 2020]. Disponível em: https://www.sc.gov.br/noticias/temas/agricultura-e-pesca/secretaria-da-agricultura-atualiza-situacaodos-cultivos-de-moluscos-em-santa-catarina

16. YouTube ${ }^{B R}$, UFPR TV [Internet]. Scientia - Maré Vermelha (07/09/15); 2015 [citado em 01 nov 2020]. Disponível em: https://www.youtube.com/watch?v=TkQGZ1WmHg8\&feature=emb_title 